\title{
Local distribution of deep centers in GaP studied by infrared cathodoluminescence
}

\author{
F. Domínguez-Adame, J. Piqueras, and P. Fernández \\ Departamento de Fisica de Materiales, Facultad de Ciencias Fisicas, Universidad Complutense, \\ 28040 Madrid, Spain
}

(Received 15 June 1990; accepted for publication 23 October 1990)

Near-infrared cathodoluminescence $(\mathrm{CL})$ in the scanning electron microscope has been used

to characterize GaP:S. Spectra of as-grown crystals show a broadband at about 1240

$\mathrm{nm}$, probably related to $P_{\mathrm{Ga}}$ antisite defects. This emission has been found to be higher at

dislocations giving a $\mathrm{CL}$ image opposite to the visible $\mathrm{CL}$ image.

Due to the use of GaP in the field of light-emitting diodes, its visible luminescence, with bands in the green and red spectral regions, has been often investigated. One of the techniques used was cathodoluminescence (CL) in the scanning electron microscope which provides information about the spatial distribution of the recombination centers. On the other side deep recombination centers in $\mathrm{GaP}$, emitting in the near infrared (NIR) have been studied by photoluminescence and optical detected magnetic resonance (OMDR) $)^{1-4}$ and several models to explain the appearance of luminescence bands in the range 1000-1300 $\mathrm{nm}$ have been given. As in the case of visible luminescence, space-resolved CL techniques could give additional information on the defects involved in the NIR emission. However, to our knowledge, such techniques have not been applied to the study of deep centers in GaP. In the present work NIR CL in the scanning electron microscope has been used to study the nature and distribution of radiative centers in liquid-encapsulated Czochralski (LEC) GaP:S.

The samples used were cut from a $\langle 100\rangle$ oriented S-doped LEC GaP wafer with a free-carrier concentration $n$ of $3-4 \times 10^{17} \mathrm{~cm}^{-3}$. The samples were observed in the emissive and CL modes in a Hitachi S-2500 or a Cambridge \$4-10 scanning electron microscope at an accelerating voltage of $30 \mathrm{keV}$ and beam currents of $10^{-7_{-}}$ $10^{-6} \mathrm{~A}$. For the obtention of panchromatic NIR CL images an optical lens was used to concentrate the light on a cooled North Coast EO-817 germanium detector attached to a window of the microscope. In some cases $C L$ images for wavelengths above $1000 \mathrm{~nm}$ were recorded by adapting a cut-on optical filter at the detector entrance. To record spectra a light guide feeding the light to an Oriel 78215 computer-controlled monochromator was used. In cases of low signals, spectra representing the average of a high number of measurements are readily obtained. The spectra, covering the range $800-1800 \mathrm{~nm}$, were corrected to include the system spectral response. Due to the low emission at room temperature, all $C L$ measurements were carried out at $170 \mathrm{~K}$. Besides as-grown samples, samples which had received the following treatments were investigated: (a) annealing in argon atmosphere at $1000 \mathrm{~K}$ for times ranging from 1 to $5 \mathrm{~h}$, (b) irradiation with $2.8 \mathrm{MeV}$ electrons to a dose of $8 \times 10^{18} \mathrm{e}^{-} \mathrm{cm}^{-2}$, (c) electron irradiation, as in (b), followed by annealings at 700 and $930 \mathrm{~K}$ for $1 \mathrm{~h}$.
Visible CL of the same samples used in this work has been previously investigated. ${ }^{5-7}$

Figure 1(a) shows the CL spectrum of an as-grown sample with a broad (full width at half maximum of 360 $\mathrm{nm})$ band centered at about $1240 \mathrm{~nm}(1.0 \mathrm{eV})$. This spectrum has been recordcd under the normal observation conditions of the scanning electron microscopy, with the electron beam focused on the sample. With a defocused beam the spectrum of Fig. 1(b) showing the tail of an intense red band and a composite broad IR band with a peak at about $1320 \mathrm{~nm}(0.94 \mathrm{eV})$ is recorded.

The $1240 \mathrm{~nm}$ band (obtained with focused beam) is strongly reduced by annealing as-grown samples above 830 $\mathrm{K}$. Annealing the as-grown samples at $1000 \mathrm{~K}$ for $1 \mathrm{~h}$ induces only minor changes in the spectra obtained with a defocused beam. The spectra show a small emission peak at $1100 \mathrm{~nm}(1.13 \mathrm{eV})$ that increases with annealing time. After $5 \mathrm{~h}$ annealing at $1000 \mathrm{~K}$, the spectrum of Fig. 2 is obtained. No spectra could be recorded with a focused beam. In the electron-irradiated samples, the NIR luminescence appears to be quenched, as previously reported for the visible range. ${ }^{6}$ Partial recovery of the emission is observed after annealing at $700 \mathrm{~K}$ and luminescence further increases by annealing at $920 \mathrm{~K}$ for $1 \mathrm{~h}$. The spectrum recorded after this treatment is shown in Fig. 3.

As described above, only the spectra of as-grown samples could be recorded when a focused beam is used. In all other cases the beam has to be defocused to get enough emission to record spectra. Consequently, NIR CL images were obtained only from the as-grown crystals. Figure

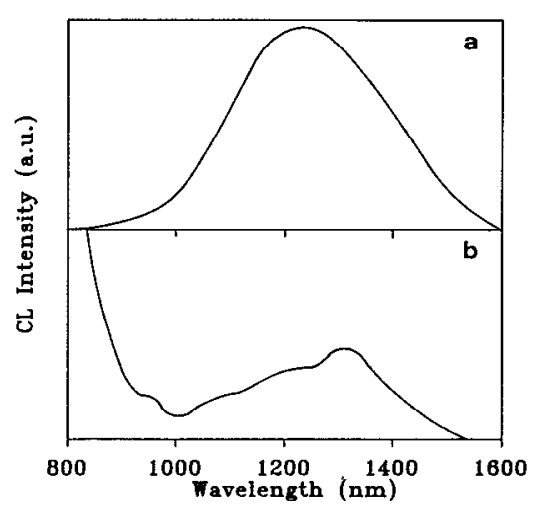

FIG. 1. NIR CL spectra from as-grown samples with (a) focused electron beam and (b) defocused beam. 


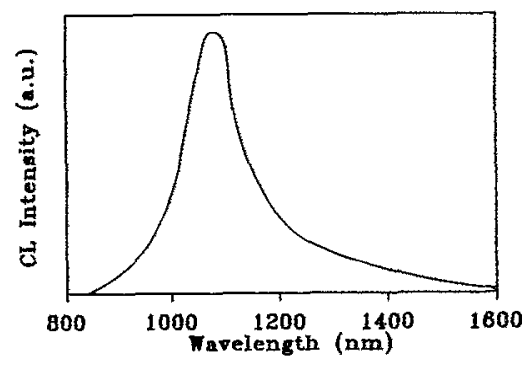

FIG. 2. NIR CL spectrum (defocused beam) of a sample annealed at $1000 \mathrm{~K}$ for $5 \mathrm{~h}$.

4(a) shows a representative CL image obtained by using an optical filter transmitting wavelengths above $1000 \mathrm{~nm}$. The corresponding image of visible CL with dot and halo contrast associated with the presence of dislocations ${ }^{5}$ is shown in Fig. 4(b). Comparison of both figures reveals that contrast in the NIR CL image is about inverse to that of the visible CL. Most of the dark dislocation points in Fig. 4(b) appear bright in Fig. 4(a).

An infrared band very close to the $1240 \mathrm{~nm}$ band observed in this work in as-grown crystals has been previously reported. Killoran et $a l^{1}{ }^{1}$ concluded from their ODMR experiments that $P_{\mathrm{Ga}}$ antisite takes part in an electron capture process which competes with the visible photoluminescence emission. In particular, they assign emissions at $1130 \mathrm{~nm}(1.1 \mathrm{eV})$ and $1275 \mathrm{~nm}(0.97 \mathrm{eV})$ to different recombination processes involving the $P_{\mathrm{Ga}}$ antisite. Yang et $a l^{2}$ have also detected the $1275 \mathrm{~nm}$ emission and have related it to the presence of $P_{\mathrm{Ga}}$. We suggest that the NIR band of our as-grown crystals is the antisiterelated band of Refs. 1 and 2 . Since in these crystals only one band is observed in the NIR region, the CL image would provide information about the $P_{\mathrm{Ga}}$ defect distribution in the sample, in particular around dislocations. The CL images indicate that the NIR emitting centers concentrate near the dislocation core and other crystal regions, leaving a denuded zone around dislocations. The fact that the $1240 \mathrm{~nm}$ band disappears by annealing would be a consequence of the annealing out of antisite defects. In $\mathrm{GaAs}$, thermal annealing of $\mathrm{As}_{\mathrm{Ga}}$ has been found to start at $780 \mathrm{~K},{ }^{8}$ which is about the temperature causing a significant reduction of the $1240 \mathrm{~nm}$ band in this work. On the other hand, annealing the as-grown samples causes the appearance of the $1100 \mathrm{~nm}$ band shown in Fig. 2. Since such

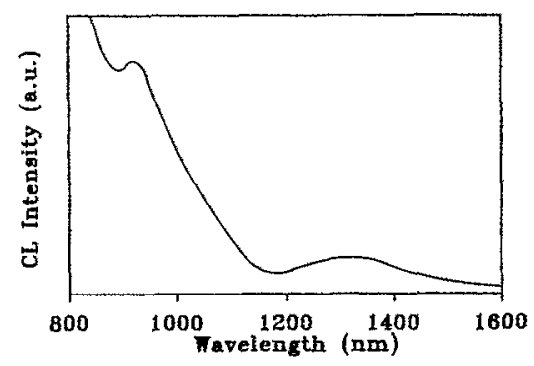

FIG. 3. NIR CL spectrum (defocused beam) of a sample irradiated and annealed at $925 \mathrm{~K}$.
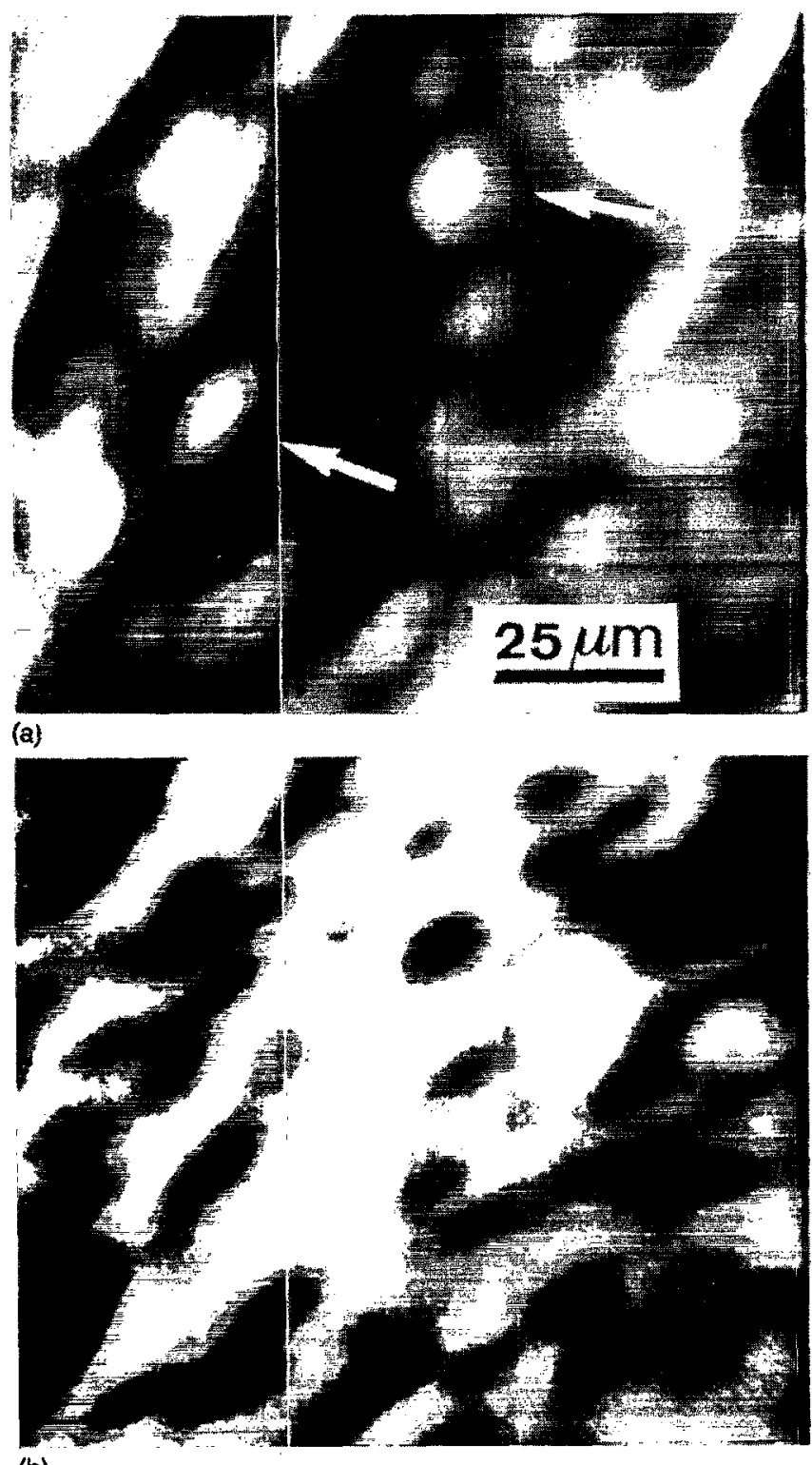

(b)

FIG. 4. (a) NIR (wavelength above $1000 \mathrm{~nm}$ ) and (b) visible $\mathrm{CL}$ inages of the same area of an as-grown sample. Arrows indicate two emergence points of dislocations.

thermal treatment causes a selective loss of phosphorus, ${ }^{9}$ vacancies in the $P$ sublattice would be involved in the emerging band. In Ref. $2, V_{P}$ have been also related to a $1100 \mathrm{~nm}$ luminescence band.

The quenching of NIR-CL by high-energy electron irradiation has been also found in the visible spectral range. ${ }^{6}$ An irradiation experiment was aimed to check if radiationinduced defects act as recombination centers in the infrared region quenching the visible luminescence. The results indicate that irradiation creates mainly nonradiative recombination centers quenching both the visible and NIR luminescence.

This work was supported by the Comision Interministerial de Ciencia y Tecnología (Project PB86-0151). The authors thank Wacker-Chemitronic (Dr. K. Löhnert) for providing the samples and Dr. P. Moser (C.E.N.G. Grenoble) for the electron irradiation. 
'N. Killoran, B. C. Cavenett, M. Godlewski, T. A. Kennedy, and N. D. Wilsey, J. Phys. C 15, L723 (1982).

${ }^{2} \mathrm{X}$. Z. Yang, L. Samuelson, H. G. Grimmeiss, and P. Omling, Semicond. Sci. Technol. 3, 488 (1988).

${ }^{3}$ M. Godlewski and B. Monemar, J. Appl. Phys. 64, 200 (1988).

${ }^{4} \mathrm{~K}$. Chino, T. Kazuno, K. Satoh, and M. Kubota, in Semi-Insulating III-V Materials (Adam Hilger, England, 1988), p. 133.

'F. Domínguez-Adame, J. Piqueras, N. de Diego, and J. Llopis, J. Appl. Phys. 63, 2583 (1988).
${ }^{6}$ F. Domínguez-Adame, J. Piqueras, N. de Diego, and P. Moser, Solid State Commun. 67, 665 (1988).

${ }^{7}$ F. Domínguez-Adame and J. Piqueras, Mater. Chem. Phys. 21, 539 (1989).

${ }^{8}$ E. R. Weber, H. Ennen, U. Kaufmann, J. Windscheif, J. Schneider, and T. Wosinski, J. Appl. Phys. 53, 6140 (1982).

${ }^{9}$ A. R. Peaker, B. Halmilton, D. R. Wight, D. Blenkinsop, W. Harding, and R. Gibb, Inst. Phys. Conf. Ser. No. 33a, 326 (1977). 